\title{
EMDR Treatment of Obsessive-Compulsive Disorder: Three Cases
}

\author{
Zoe Marsden \\ Leeds Improving Access to Psychological Therapies Service, United Kingdom
}

\begin{abstract}
This article reports on the first 3 randomly allocated cases treated by the author in an ongoing trial comparing eye movement desensitization and reprocessing (EMDR) with cognitive behavioral therapy (exposure and response prevention) in the treatment of obsessive-compulsive disorder in a U.K. primary care setting. This article describes the treatment and data collection procedures, followed by a summary of each of the 3 cases supported by quantitative and qualitative data. The Adapted EMDR Phobia Protocol (Marr, 2012) was provided, following the trial protocol of 1-hour, 16-session treatment. The Yale-Brown Obsessive Compulsive Scale was administered at every 4th session. At posttreatment, 2 of the 3 cases showed more than a $50 \%$ reduction on validated psychometric measures, with symptoms below diagnostic cutoff. The final case started treatment below the diagnostic cutoff on the primary outcome measure and showed a slight improvement. Six-month follow-up data showed maintenance of treatment effects. Transcripts from a semistructured telephone interview carried out by an independent researcher following treatment were analyzed using a 6-stage thematic analysis method, which identified 3 themes: the role of traumatic experiences, role of shame, and importance of therapeutic alliance. This article concludes with a discussion of implications for EMDR practice and theory.
\end{abstract}

Keywords: eye movement desensitization and reprocessing (EMDR) therapy; obsessive-compulsive disorder (OCD); randomized controlled trial; Adapted EMDR Phobia Protocol; mixed methods; case study

0 bsessive-compulsive disorder $(\mathrm{OCD})$ is the 4 th most common mental disorder and is among the top 10 most disabling illnesses described by the World Health Organization in terms of lost income and decreased quality of life (Veale $\&$ Wilson, 2005). It is a psychological condition associated with recurrent obsessive thoughts and/or compulsions that interfere negatively and substantially with daily functioning. People are unable to control either the thoughts, such as harm coming to a loved one, or the compulsions, such as handwashing, counting of things, or checking if a door is locked. These behaviors or mental rituals are aimed at preventing distress and preventing some dreaded situation or event. Diagnosis is based on the symptoms taking up large amounts of time and impinging on daily activities. OCD affects $1 \%-2 \%$ of the population (Kessler et al., 2005). It is unusual for symptoms to start after the age of 35 years, and half of adults with OCD develop problems in childhood.

Cognitive behavioral therapy (CBT) based on exposure and response prevention (ERP) is the most usual psychological treatment recommended for OCD. ERP is a behavioral therapy that was first developed by Victor Meyer, a U.K. psychologist in 1966. ERP involves repeated exposure to distressing situations or cues (e.g., objects perceived to be contaminated) while preventing the use of ritualized or repetitive behaviors (e.g., handwashing) that are used to neutralize distress or to relieve obsessive preoccupations (e.g., fear of becoming contaminated and ill). ERP is effective at reducing the intensity of OCD symptoms for about $50 \%-60 \%$ of patients who complete treatment (Fisher \& Wells, 2005). Although there is considerable evidence in support of ERP (Abramowitz, 1997, 1998; Foa, 2010; Franklin $\&$ Foa, 2002), it has been argued that exposure tasks in session and as homework can be difficult to tolerate, patients often find it too frightening to face their worst fears, and this leads some clients to drop out of treatment (Maher et al., 2010). It is estimated that $25 \%$ of patients drop out of treatment (Aderka et al., 2011). 


\section{EMDR}

Difficulties completing ERP and dropout rates raise a question about how to meet the needs of those for whom ERP is less effective. There is some recent evidence that eye movement desensitization and reprocessing (EMDR) therapy maybe another useful treatment option for this condition. EMDR (Shapiro, 2001) is a well-established and empirically supported treatment for posttraumatic stress disorder (PTSD; American Psychiatric Association, 2013). There are approximately 20 randomized controlled trials (RCTs) supporting its efficacy for PTSD, and various meta-analyses have found EMDR to be equivalent in effect for PTSD as cognitive behavioral approaches (Gerger et al., 2014; Watts et al., 2013). Over the years since Shapiro developed the standardized EMDR procedures, numerous protocols have been developed for specific disorders or situations. Protocols developed for OCD include Logie and de Jongh's (2014) "flashforward procedure" as well as Marr's "Adapted EMDR Phobia Protocol” (Marr, 2012), which was used in this study.

EMDR therapy uses a structured eight-phase procedure that begins with history taking and preparation. In Phase 3, the visual, cognitive, affective, and sensory components of the targeted memory are identified, and clients rate the validity of the preferred self-referring positive cognition on the Validity of Cognition (VOC) Scale (where $1=$ not true, $7=$ completely true) and the level of emotional disturbance on the Subjective Units of Disturbance (SUD) Scale $(0=$ no disturbance, $10=$ worst possible disturbance). In Phase 4 , the client recalls the targeted memory while simultaneously engaging in sets of eye movements (or alternating bilateral audio and/or tactile stimulation). Phase 4 continues until the distress level, measured by the SUD scale is 0 . In Phase 5, the positive cognition is strengthened using further eye movements until the VOC score is 7. Phase 6 is considered completed when a client can bring the memory and positive cognition to mind without any body tension. Phase 7 is a careful closure of the session including use of self-care techniques, and Phase 8 is the reevaluation that takes place at the start of every subsequent session.

\section{EMDR and Obsessive-Compulsive Disorder Treatment}

There are several case studies providing preliminary evidence that EMDR therapy may be an effective way to treat patients with OCD. Keenan, Keenan, Ingham, and Farrell (2014) described a series of eight cases who had received CBT within the last 5 years but were still experiencing OCD symptoms. The standard EMDR protocol was used to resolve past aversive life experiences or intolerance of uncertainty for those reporting no past aversive life experiences. All cases showed significant improvement at 3-month follow-up. Bekkers (as cited in Bohm \& Volderholzer, 2010) described significant symptom reductions in four out of five patients with OCD treated with EMDR adjunctive to ERP. Bohm and Volderholzer (2010) described the effects of ERP and EMDR therapy in three OCD cases, although again the combination of therapies makes it difficult to assess the unique contribution of EMDR. Nazari, Momeni, Jariani, and Tarrahi (2011) compared EMDR with citalopram in the treatment of OCD in Iran. This was a randomized trial of 90 patients, and it concluded that both therapeutic methods had significant effects, although EMDR appeared to be more efficacious in the short term. In his paper describing four OCD clients treated with EMDR, Marr (2012) used two slightly different adaptations of Shapiro's phobia protocol (Shapiro, 2001). All participants showed significant symptom reduction posttreatment and at 4- to 6-month follow-up. One of Marr's adaptations, the Adapted EMDR Phobia Protocol, was used in this study.

\section{Marr's Adapted EMDR Phobia Protocol}

In Shapiro's standard EMDR protocol, memories are processed using a three-pronged approach (Shapiro, 2001), working first on distressing past memories, then current triggers, and finally addressing a future template of adaptive future action. In Marr's (2012) Adapted EMDR Phobia Protocol, treatment starts with the current triggers (OCD obsessions and compulsions) followed by the future template (imagining successful future action) and then past-related disturbing memories (if any). Clients are actively involved in deciding the order in which the current triggers (OCD thoughts and behaviors) and future templates are worked through in treatment. Notably, the client is directed to start processing with a target image, emotion, associated body sensation, but not negative or positive self-referencing cognitions. Work on cognitions, the positive cognition, only takes place when the current future and past phases have been completed. Marr's rationale for altering Shapiro's standard three-pronged approach is that

Although OCD may have originated in early experiences... [he sees $\mathrm{OCD}$ ] as a self-maintaining disorder ... [and] is best understood as a series of self-perpetuating and interlaced traumatic events ... [that] reinforce and perpetuate multidimensional disturbing patterns of thoughts and behaviours. (p. 11) 


\section{Current Study}

Given the growing interest in EMDR as a possibly helpful alternative to CBT/ERP interventions, the purpose of this article is to present a series of three OCD cases treated by a single therapist applying one of the therapy protocols developed by Marr (2012). As such, this article offers an independent assessment of this protocol, applied within the context of a pragmatic feasibility RCT that is currently underway (http:// www.controlled-trials.com/ISRCTN16396325). The case series includes the first three completed cases that were treated by the author and for whom pre- and posttreatment clinical outcome measures and posttreatment qualitative interviews were available. This article initially describes the treatment and data collection procedures, followed by a summary of each of the three cases supported by qualitative and quantitative data. Finally, the article concludes with a discussion of implications for EMDR practice and theory.

\section{Method}

\section{Setting and Context}

Cases described in this article were recruited to a pragmatic trial conducted in a primary care mental health service in Northern England aligned to the national Improving Access to Psychological Therapies (IAPT, 2011) program. IAPT services in England deliver evidencebased psychological therapies for common mental health problems as part of a "stepped care" model that offers progressively more intensive therapies for patients who do not make improvement at the earlier steps of care. Patients receiving psychological therapies are seen for treatment in general practitioner (GP) surgeries, health centers, or community-based centers.

The primary objective of the trial was to examine the feasibility of recruiting, randomizing, and completing structured treatment protocols for EMDR and CBT (ERP) with patients with OCD and measuring outcomes at repeated intervals. Patients were eligible to participate in the trial if they were consenting adults older than the age of 17 years, were suitable for treatment in a primary care setting, met Diagnostic and Statistical Manual of Mental Disorders (5th ed.; DSM-IV) diagnostic criteria for OCD established using the Mini-International Neuropsychiatric Interview (MINI) schedule (Sheenan et al., 1998), and this was the person's primary concern or reason for seeking treatment. Exclusion criteria included not being suitable for treatment in a primary care setting because of acute suicidal risk, psychosis, or alcohol or drug dependence, and OCD not being the patient's primary concern for treatment (Delgadillo, Marsden, Lovell, Blore, \& Ali, 2014).

\section{Recruitment and Data Collection}

Consistent with the trial procedures, the cases described in this article were screened by mental health practitioners at time of entry to the service, and based on a probable diagnosis of OCD, they received study information leaflets and were invited to have a telephone diagnostic interview with a researcher. Once an OCD diagnosis was confirmed using the MINI OCD module, consenting cases were randomly allocated to participating therapists by a research administrator. Hence, the three cases presented in this article were randomly allocated to the author.

A set of patient outcome measures was collected as part of the clinical procedures and wider trial. The self-completed Yale-Brown Obsessive Compulsive Scale (YBOCS) is a brief 10-item measure of OCD symptoms that renders a severity score between 0 and 40 (Goodman et al., 1989). A diagnostic cutoff of 16 and symptom reduction of $50 \%$ or more has been recommended to assess reliable and clinically significant changes (Tolin, Abramovitz, \& Diefenbach, 2005). The Obsessive Compulsive Inventory (OCI) is a 42 -item questionnaire covering seven domains: washing, checking, doubting, ordering, obsessing, hoarding, and mental neutralizing (Foa, Kozak, Salkovskis, Coles, \& Amir, 1998). Convergent validity has been demonstrated by researchers who have explored correlations between the OCI and YBOCS scales (Foa, Kozak, Salkovskis, Coles, \& Amir, 1998). The brief YBOCS measure was administered at baseline and every fourth treatment session and the OCI at baseline and final session.

Semistructured qualitative interviews carried out following treatment featured in this article were conducted by another member of the research team to minimize social desirability bias using a semistructured interview topic guide designed for application in a clinical trial (Delgadillo et al., 2014). The topic guide asked questions about the name of therapy accessed, what the treatment involved, aspects of treatment that were helpful or unhelpful, changes in symptoms posttreatment, how well the treatment worked, and how it can be improved. Interviews were conducted via telephone, audio recorded, and later transcribed verbatim.

\section{Data Analysis}

In this article, a narrative description of each case is presented first. Pseudonyms are used instead of clients' actual names to protect their confidentiality. 
Descriptive statistics are presented alongside cases to illustrate changes in validated outcome measures during the time of the intervention. No formal statistical tests were applied given the small number of cases. Transcripts from qualitative interviews were interpreted by the author applying a six-stage thematic analysis method (Braun \& Clarke, 2006). Data analysis was carried out initially by searching for meanings and patterns in the interview transcripts (Phase 1). Next, open coding was generated for all data extracts (Phase 2). In Phase 3, data extracts were organized into potential emerging themes. In Phase 4, themes were reviewed to develop a thematic map with linked codes and extracts across the data set. Phase 5 involved defining and naming each of the themes, whereas Phase 6 involved producing a brief report, selecting extracts that adequately represent the themes.

\section{Case Study 1: Abigail}

\section{Current Life Context}

Abigail was an 18-year-old woman in her first year of university. She had a large group of friends whom she spent time with and some very close friends. She spent the week living in the halls of residence and the weekends staying with her mother and brother aged 12 years, returning to university on Sundays after working at a part-time job. She described night time checking behaviors as "tedious" that she had struggled with since around the age of 9 years. Abigail said no one in her family had OCD but that she noticed that her little brother was starting to check things and this concerned her. Abigail described a happy childhood, with her parents separating 3-4 years ago, which she found difficult at the time but said did not exacerbate her OCD symptoms.

\section{Clinical Presentation}

The checking behaviors at night included checking doors, windows, plug sockets, ornaments, and duvet corners; making sure clothes were in drawers; having certain coats hanging on the back of her door; and so on. Abigail tended to check in multiples of three. On a "good night," the checks would take half an hour, but on a "bad night" (usually when Abigail knew she had to be up early), up to 5 hours. The checking was clearly having an impact on Abigail's ability to concentrate and study, and she was often very tired. Her goal for treatment was to be able to go to bed and sleep without having to engage in a checking routine. Abigail was planning to move into a shared house in her second year of university and was concerned how she would manage this with her OCD behaviors, which was her reason for seeking treatment at this time.

Abigail described also struggling with driving in the dark when she would repeatedly check whether her headlights were on by turning them off and on. Abigail said she did well academically and tended not to worry about things like exams. She described liking to feel in control (or to avoid anxiety), and as a result, she rarely drank alcohol (and did not take any illicit drugs) in case her drink got spiked (this had never happened). She did not like flying or being a passenger in a car.

Abigail was not taking any antidepressant medication and previously had some CBT at age 12 years with the child and adolescent mental health services which she remembers as unhelpful. There were no identified risk issues such as suicidality or risk to others. At the time of her first EMDR session, Abigail completed YBOCS and scored 24 and OCI at 34.

\section{Description of EMDR Process}

During the initial client history taking phase, Abigail described a past memory when she was approximately aged 9 years. She recalled watching a horror film called The Ring with friends during a sleepover. She said with hindsight she did not understand why her parents allowed her to watch the film because it was "so scary." She remembered being very scared of the "little girl that comes out of the TV and plug sockets to kill people." As a teenager and adult, Abigail enjoyed watching horror films and had watched The Ring again and "didn't find it scary at all." No other memories emerged during history taking. Abigail was not able to identify any obsessive thoughts that occurred to her before her checking behaviors took place during evenings but said she knew generally her concerns were about safety of herself and others. She also said she rarely felt anxious prior to her checking behaviors at night because she "would not resist in any way, wanting to get the checks done so she could sleep."

The preparation phase of EMDR focused on selfcare techniques and building therapeutic alliance. Abigail initially struggled to identify any feelings of anxiety or discomfort in the body. However, after working on the calm place exercise (Parnell, 2007) and the resulting feeling of lightness this gave her, she was able to differentiate this, realizing that feeling of anxiety in her chest and head felt normal to her.

Sessions 3-6: Processing Current Triggers. Abigail chose to start working on the current trigger of her night time checking behaviors. Abigail began by 
playing an imagined scene-by-scene videotape of her night time checking behaviors. When she became aware of any feelings of any affective or somatic distress, she would stop the tape for processing to take place. During processing, TheraTappers were primarily used to provide alternating bilateral stimulation. Abigail stopped the tape on three occasions because of experiencing distress when desensitization took place. A further past memory came to mind of the first time she may have checked at night, being around age 9 years and was sharing a bed with her little brother aged 2 years. She remembered he was asleep and she got out of bed to check doors to make sure he would be safe.

The procedure was repeated to get to the point where Abigail could run the tape twice in her mind without any distress as per protocol. After two sessions, Abigail reported she was feeling frustrated with the treatment because she was not seeing any progress outside of sessions. As a result, I suggested switching to working on the current trigger of driving checking behaviors, which hopefully could be more easily contained. The imagined videotape was of the last time Abigail had checked her headlights while driving. Abigail was quickly able to run this through with no distress. A further past memory surfaced from some years ago, of reading on Facebook a message to pass onto others "that gangs were flashing other cars with their headlights to mark a car out as pray to other gang members, leading to death."

After this session, Abigail reported partial remission of her checking behavior while driving. We then returned to the current OCD trigger of the night time checking routine. After one stop to process distress, Abigail was able to run through the mental videotape twice without any distress. At the beginning of the subsequent session, Abigail reported a slight decrease in night time checking behaviors.

Sessions 7: Future Templates. The work then moved on to the future templates (imagining successful future action; preferred emotion-strength, positive cognition-I am safe) for the night time checking behaviors and checking of headlights while driving. This was accomplished without any challenges with Abigail describing feeling stronger and more confident.

Sessions 8-9: Processing Past-Related Memories. Past-related memories were targeted using the standard EMDR protocol, starting with watching the horror film at age 9 years. In Phase 3 of the standard protocol, Abigail's negative cognition was "I am unsafe" with the corresponding positive cognition "I am safe," emotion fear, and she felt this bodily in her chest and head. During processing, Abigail quickly realized she had not watched The Ring at the sleepover event but had actually watched Scary Movie 3 with her friends instead. She then reflected after further desensitization that she now knew her parents had watched The Ring with some neighbors, and she had overheard them talking about it. Phase 4 was completed to no affective or somatic distress, and the image of the girl in The Ring changed from a black and white image to colored. Abigail said the image was no longer distressing (SUD 0). In Phase 5, the positive cognition changed to "I am over it" which strengthened until feeling completely true, and Abigail was no longer able to bring the image of the girl from the film to mind. The body scan revealed no residual tension, and on closing the session, Abigail said she felt relieved and impressed with what her mind had done.

In the next session, other identified past memories were processed quickly. By this point, Abigail had reported a reduction in her checking behaviors lasting only 15 minutes at night, saying it was easier to just continue doing some "little checks as it was such a habit" but reporting feeling no anxiety. With some psychoeducation and discussing the pros and cons of continuing the checking, Abigail agreed to a plan to stop her checking behaviors after her exams which were the next week. The plan for this incorporated being both at university and at home.

Sessions 10-11: Maintenance. After her exams, Abigail reported no checking at all for 10 days while at university after which she had spent a few days at home. On the first night at home, she did not check although she had some thoughts to check, which she was able to easily ignore. The second night at home, she had almost completed her checking behaviors before she had realized she was doing them. However, Abigail's beliefs about her checking had changed, and she no longer said it was "easier to do a quick check" and instead described feeling strong and proud about this change.

The final session reviewed progress. Abigail reported no further checking behaviors. The thought to check did happen sometimes, but she was able to dismiss this easily. She reported sleeping well and generally feeling more relaxed, had started going out to clubs drinking with her friends, and was able to get up in the middle of the night to use the toilet if she wanted to (she would not have done this previously as would need to "check" before getting back to sleep). OCI at final session scored 0. The YBOCS was not completed at final session (Session 11) as was being scored every fourth session as per trial protocol. YBOCS at 6-month follow-up scored 0. 


\section{Case Study 2: Sarah}

\section{Current Life Context}

Sarah was a 40-year-old woman who lived with her husband and 2-year-old son and worked part-time in a managerial/administrative role for a public organization. She described struggling with OCD symptoms for the last 20 years. Sarah said she thought the difficulties may have started when living in shared housing as a student. She remembered on a few occasions housemates forgetting to lock the front door but did not find this distressing. Sarah described no family history of OCD or any other mental health issues. She described a good support network of family and friends but said she had only spoken to two friends briefly about her OCD over the years that she knew also struggled in a similar way and her husband was aware. She had no previous treatment for OCD was not on any medication, and there were no identified issues with risk, illicit drug use, or alcohol.

\section{Clinical Presentation}

Sarah described checking behaviors when leaving the house, checking that all the lights were off, door handle, and cooker, all at least three times while saying to herself "it's off, it's off," or "it's locked, it's locked." At bedtime, Sarah would check if her husband had locked the door a few times by asking him about it and would check the cooker and lights. Sarah would go into her son's room before bed to check if there was nothing dangerous or unusual in his cot. If out shopping, Sarah would check if credit cards were back in her purse and would check them again before bed. When going swimming with her son, she would check if their bag was in the locker three times. Sarah could not identify an obsessive thought that preceded the checking behaviors but knew she was concerned about safety/responsibility. Her goal of treatment was to be able to "look at something, know it's off, and walk away with no anxiety." At her first EMDR session, Sarah completed YBOCS and scored 10 and OCI 33, both mild scores.

\section{Description of EMDR Process}

The preparation phase focused on self-care techniques, where a calm place was established, as well as practicing a breathing technique, which Sarah reported finding very helpful throughout the treatment sessions.

Sessions 4-7: Processing Current Triggers. Sarah chose to start working on the current trigger of getting ready for bed and began by running an imagined videotape of the checking behaviors in her mind and when she became aware of any feelings of any affective or somatic distress would stop the tape for processing to take place. During processing, TheraTappers were primarily used to provide alternating bilateral stimulation. The number of times Sarah needed to stop the tape to process distress reduced gradually over two sessions until the imagined videotape was run through without any distress. Because no past distressing memories were arising or had been identified during the initial client history, the floatback technique (Shapiro, 2001) was used, where the therapist guides the client to recall past events with similar thoughts, affective, and somatic distress. However, no past memories emerged.

Sarah reported working very hard between sessions to resist the urge to check which she did with some success, reducing checking behaviors to once or twice. This was not set as homework but was independently motivated by the client. All other current triggers were processed. Within the context of supporting Sarah's own hardworking approach to overcoming her problems, it was suggested to try outside of session to "go into her body more" and notice the body sensations and emotions passing in EMDR style to "let whatever happens happen" rather than more cognitively trying to resist the urge to check.

Sessions 8-9: Future Templates. The work moved onto the future templates (imagining successful future action) for leaving the house, bedtime routines, and other current triggers with Sarah reporting feeling more in control of her checking behaviors. We attempted to work on the past memory (of housemates leaving doors open/unlocked) which had been identified at the initial client history phase. However, just as Sarah had said at the beginning of treatment, she did not feel any distress when recalling this memory and found it at best a vague memory.

Sessions 10-12: Maintenance. In two further sessions to review progress, Sarah said her checking behaviors were better but not resolved, that she felt more in control and was putting less pressure on herself. Future templates were repeated for leaving the house and bedtime routine at Sarah's request because she said she found this helpful and a relapse plan developed.

At the final session, YBOCS had reduced to 7 , a reduction of 3 points, and OCI had reduced to 16 . YBOCS at 6-month follow-up scored 8.

\section{Case Study 3: Jan}

\section{Current Life Context}

Jan was a 50-year-old woman who lived with her partner and worked part-time as an administrative assistant. 
She had previously worked as a lawyer for 25 years until being made redundant in 2013. Jan said she had hated the job, found it very stressful, and being made redundant very upsetting. She was close to her parents for whom she now played a caring role and described that over the years she had let friendships drop and had no friends. Jan had several health problems: chronic cystitis (getting up two to three times a night to use the toilet), irritable bowel syndrome, and eczema.

\section{Clinical Presentation}

Jan described a happy childhood where she worked hard, was head girl, and was exam-driven. She remembers her grandfather having checking behaviors and watching him from the car going back to check if the door was locked. Jan said she thought her OCD started in her early 20s when she started working as a lawyer and described an incident when she was late for a client function, which she still found distressing when she thinks about it. Jan described herself as very thorough, detail-focused, can sometimes find it difficult to finish a job, a worrier, can't fly or drive on motorways because of anxiety, and said she likes to be "well-organized, clean, and in control." She said that she enjoyed gardening and walking with her partner but that housework fills her time.

Jan described several checking behaviors: on leaving the house; at bedtime; some hoarding (could not throw out papers or magazines as needing to check if missed something); struggling to lock up the building at work (one of her duties); and some issues related to contamination, excessive hand washing, and not touching door handles outside of her home. Jan was finding that her OCD was significantly affecting her ability to function day to day. At her first EMDR session, Jan completed YBOCS and scored 17 and OCI 63. Jan was on no antidepressant medication, had no previous treatment for OCD, and there were no risk, illicit drug use, or alcohol issues. She had accessed treatment at this time because she was no longer able to lock up at work because of her checking behaviors and was concerned that she may lose her job. Keeping her job was her main goal of therapy.

Description of EMDR Process. In the preparation phase, a calm place was established and light stream technique (Shapiro, 2001) was used with good effect. Jan had previously attended a brief mindfulness course and was encouraged also to use techniques learned previously.

Sessions 4-10: Processing Current Triggers. Jan chose to start with working on the current trigger of not touching door handles. During processing, TheraTappers were primarily used to provide alternating bilateral stimulation. Jan exhibited high anxiety levels while running the imagined videotape and subsequent processing. Based on Jan's feedback, it became clear toward the end of the session that she was not running the tape based on how she was managing her OCD routine currently (using her elbow to open the door) but rather based on how she wanted to do this in the future (using her hand to open door handles). At the end of the session, Jan reported being surprised how she could tolerate such high levels of anxiety which she then noticed fading away and I complimented her on her strength and resilience.

Jan reported that the previous session had "stirred things up" for her, she felt different about door handles, but her behavior had not changed. She felt she had dealt with stress differently in the last week, saying she had felt angry and then it had passed. Over the next few sessions, work continued on the current triggers with Jan reporting back at the beginning of each session that she was feeling more relaxed, calmer, and reflective. There was some reduction in OCD symptoms, although Jan reported further OCD behaviors not discussed previously at history taking, which she had not previously considered to be symptoms of her OCD but now realized that they were.

Sessions 11-12: Future Templates. Given the number of current triggers and the restriction of 16 sessions because of the research protocol, it was agreed to end the work on the current triggers and move onto working on the future templates. It was hoped that given the progress Jan had made outside of session and the generalizing effect that can happen with EMDR, not completing all the current triggers would not impact negatively on recovery. While working on the future templates, Jan told me there was a past distressing memory which she found very difficult to talk about. She said in 1986, her brother had a major crisis in his life which she had to deal with because her parents were on holiday. She had never told anyone about this time in her life, not telling her partner and had never talked about it since with either her parents or her brother. She described feeling incapable of dealing with the situation, feeling out of control, and the memory remained very distressing and something she could not talk about in any further detail. I suggested we could work on this memory using the blind to therapist (B2T) protocol (Blore \& Holmshaw, 2009), which is a method of processing a distressing memory without the client disclosing the trauma content, which Jan agreed to. 
Sessions 13-15: Processing Past-Related Memories. This past-related memory was targeted using the B2T protocol. At one point, processing became stuck-in other words, there was no change in either the image, thoughts, emotions, or body sensations. After trying the usual strategies, the floatback technique (Shapiro, 2001) was used. A memory surfaced approximately aged 7 years, when on holiday in a caravan, with Jan being upset talking to her mother. At Jan's request, she returned to process the initial memory targeted, and by the end of the session, Jan reported starting to feel calmer and spontaneously moving toward a positive cognition of "I did my best." In the following session, the distressing memory from aged 7 years was targeted with negative cognition of "I am to blame" and feelings of shame that were felt in the chest. This memory was processed to an SUD of 0 , no distress. Phase 5 was completed with the positive cognition of "I'm okay" and the body scan being clear of any residual tension. Jan then returned to work on processing the initial memory using the $\mathrm{B} 2 \mathrm{~T}$ protocol and was able to reach an "SUD" of 0 , no distress. In Phase 5, Jan changed her positive cognition to "I am strong," which strengthened past a VOC of 7.

In Session 15, the distressing memory which Jan reported in history taking (just starting work as a lawyer) was targeted and completed to an SUD of 0 , with the positive cognition being "I am strong."

Sessions 16-17: Maintenance. The final session consolidated the work, working on a relapse plan. At a review session 4 weeks later, Jan had maintained her progress, she felt the OCD was "receding naturally" and said she felt a lot more positive, able to lock up at work and working more hours, and that her life was changing for the better. Her husband had noticed she was a lot happier and much lighter in mood. At Session 16, the YBOCS had reduced to 6 and OCI to 4 . YBOCS at 6 months follow-up scored 6 .

\section{Results}

The results of the patient outcome measures and the thematic analysis of posttreatment semistructured qualitative interviews suggest that overall the treatment worked well and was well received by the three clients involved.

\section{Patient Outcome Measures}

Case Study 1 shows a significant reduction in OCI scores from 34 at baseline to 0 at the final session. YBOCS score at baseline was 24; it was not completed at the final session (Session 11) because it was being scored every fourth session as per trial protocol. YBOCS at 6-month follow-up scored 0 . Similarly with Case Study 3, YBOCS scored 17 at baseline, 6 at the final session, and 6 at 6 -month follow-up. OCI scored 63 at baseline and 4 at the final session. Both these cases demonstrate reliable and clinically significant changes with symptom reduction of more than $50 \%$ and below diagnostic cutoff of 16 on the YBOCS. Case Study 2 started treatment below diagnostic cutoff on the YBOCS and showed only a slight improvement, scoring 10 at baseline, 7 at the final session, and 8 at 6-month follow-up. OCI scored 33 at baseline and 16 at the final session, showing just more than $50 \%$ reduction in symptoms (see Figures 1 and 2).

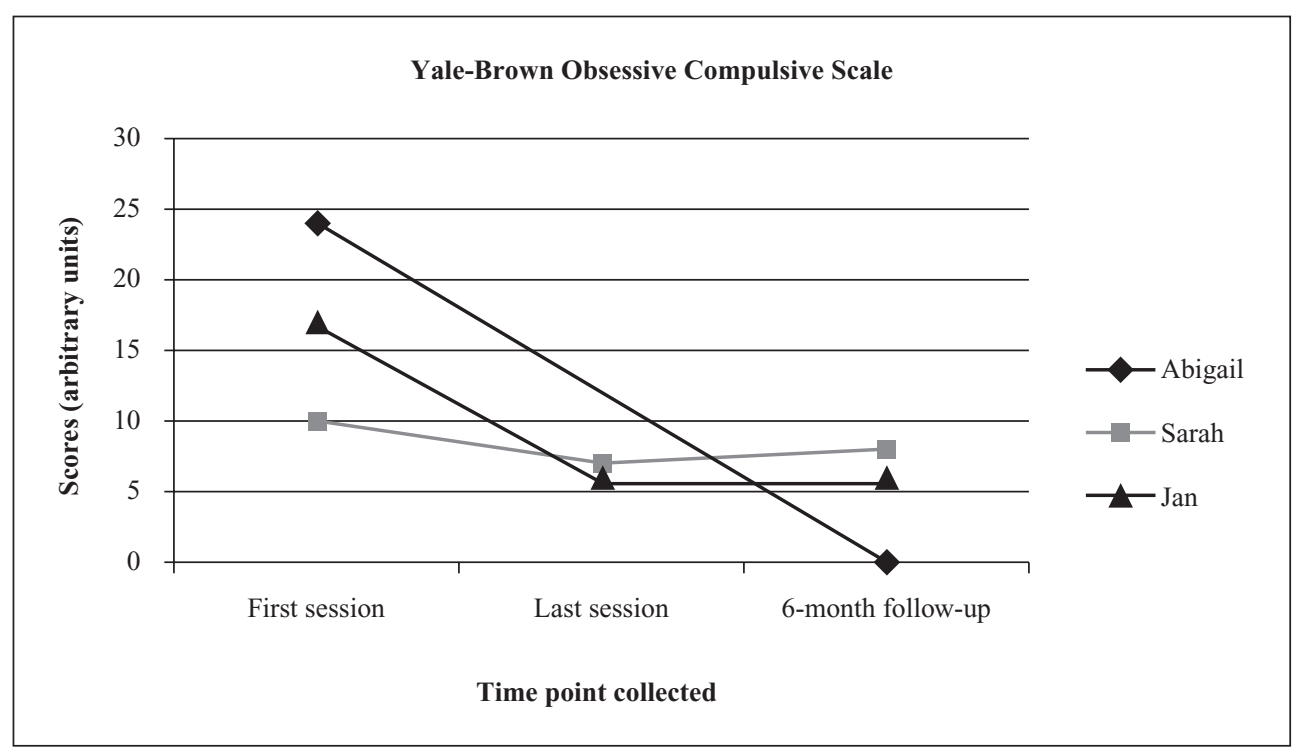

FIGURE 1. Yale-Brown Obsessive Compulsive Scale questionnaire scores collected at first and last session and 6-month follow-up. 


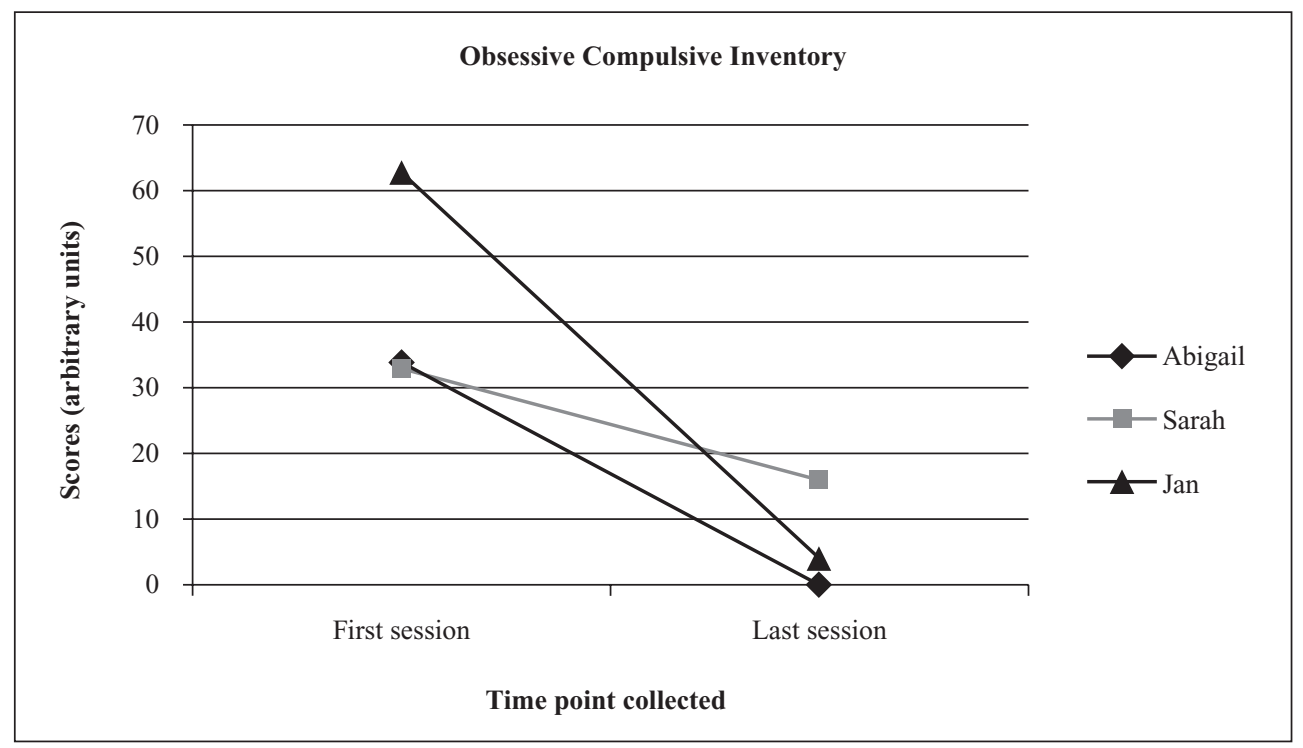

FIGURE 2. Obsessive Compulsive Inventory questionnaire scores collected at first and last session.

Participants mentioned the benefit of treatment during the semistructured interviews. Both Sarah and Jan talked about increasing their understanding of the OCD during the treatment, with Jan realizing that she had more OCD symptoms than she originally thought. Abigail and Sarah both said they found the self-care techniques helpful. The only negative evaluation of the treatment came from Abigail who found working on the current triggers of her OCD "frustrating." On the other hand, Abigail also described the therapy as "life changing" with her "symptoms gone" and also reported a generalizing effect of treatment, whereby it "helped general anxiety" and "got strength from the sessions" . . . and "new found confidence." Jan also described feeling "much less stressed generally ... staying calm ... surprising just how much less stressed I am." Sarah described "feeling more in control" and found the sessions helpful because she could talk about "feelings" and "develop coping strategies."

\section{Thematic Analysis}

Transcripts from qualitative interviews were interpreted by the author applying a six-stage thematic analysis method (Braun \& Clarke, 2006). This revealed three key themes: role of traumatic experiences, role of shame, and importance of the therapeutic relationship.

Role of Traumatic Experiences. Sarah described being unable "to uncover the root cause of [my] OCD," "one of the challenges" of the treatment. She said she does not "know why couldn't . . . don't think blocking it, just can't remember." For the other two participants, the past-related memories seemed a significant factor in the treatment. For Jan, it was a "shock to go back and identify past occasions not dealt with," although the "distress [was] contained to the sessions" and "never thought events in my past had any connection" and remembered the therapist helping her "from one past event" to a "prior past event" (floatback technique). Abigail described the "flushing out of past memories," "felt like stuff shifted in the brain," and "one session then just gone."

Role of Shame. Jan described memories that "I have never spoken to anybody about" that were "key" in her treatment. All three participants commented on the benefit of being able to talk about their OCD symptoms during their treatment with Sarah saying, "It felt good to be able to talk about it."

Importance of Therapeutic Alliance. All three participants talked about the positive impact of the therapist, in other words, the therapeutic alliance developed. Both Abigail and Sarah described the therapist as "easy to talk to," with Sarah saying she "felt safe" and Abigail saying she "felt comfortable" and the therapist "very accommodating." Jan described the therapist as "wonderful."

\section{Discussion}

As with other previously published cases using EMDR with clients with OCD, these three case studies show positive results and hopefully add to the discussion on the most effective EMDR approach to help this client group. The author found the Marr (2012) protocol easy to follow in practice. Trying to estimate how many 
sessions were needed for each phase of treatment was more difficult, particularly because of the trial restriction of 16 sessions, although session restrictions also apply in routine practice. It is the author's experience that it is possible to bypass working on all current triggers identified because of time restrictions, as long as some progress has been made, there appears to be the generalizing effect that can be seen with EMDR. It was also found helpful to avoid use of "cognitive" interweaves given this particular client group's propensity to analyze. Marr's protocol does not address cognitions until working on any past-related distressing memories toward the end of treatment, which was found to be helpful in this regard. Another positive of the protocol for the author was that it felt very client-centered.

\section{The Role of Trauma Memories}

The adaptive information processing model of EMDR puts forward that psychological symptoms are often as a result of past unprocessed traumatic material. Shapiro writes of these being either "small $t$ or big T" traumas which can lead to PTSD-like symptoms or other anxiety disorders (Shapiro \& Forrest, 2004). The role of trauma in the etiology of OCD has been explored with a significant relationship noted between OCD symptoms and one or more traumatic life events (Cromer, Schmidt, \& Murphy, 2007; de Silva \& Marks, 1999; Rosso, Albert, Asinari, Bogetto, \& Maina, 2012). Several studies have highlighted the emergence of both PTSD and OCD following combat or other violent trauma (Fostick, Nacasch, \& Zohar, 2012) and both share several common symptoms. PTSD and OCD involve unwanted intrusive thoughts, avoidance behavior directed by the need to avoid the cue that causes the distress, and negative thoughts $/ \mathrm{mem}$ ories which elicit intense anxiety (Fostick et al., 2012).

In the case studies outlined in this article, the case to show the least improvement was Sarah (3 points reduction on YBOCS) whose initial score on the YBOCS was below diagnostic cutoff and who identified no traumatic memories. In contrast, Abigail came to therapy with an already developed hypothesis that the horror film she thought she had watched aged 9 years triggered her OCD. During her qualitative interview following treatment, Abigail indicated that processing this memory was the turning point in her recovery. Similarly, Jan indicated past traumatic memories were a surprising and significant part of her treatment.

During the first phase of the OCD protocol (current triggers) with Abigail, a further distressing memory emerged related to her checking behaviors when driving. This case fits well with the description of the progression of OCD given by Fostick et al.
(2012) with OCD symptoms initially being related to the trauma-in Abigail's case, the horror film ("little girl coming out of plug sockets," etc.) initiating related checking behaviors and then becoming "independent and generalized" (checking whether headlights were turned on during night time driving). Here a distinct trauma memory, a "small $t$ " trauma surfaced as precipitating this particular OCD behavior.

It appears that this is one of the advantages of changing Shapiro's standard EMDR protocol (with its three-pronged approach of first processing past distressing memories, then processing current triggers, and finally the future template) to Marr's adapted protocol, that these distinct traumas can arise relating to different current triggers (obsessions and compulsions). Conversely, when clients are able to identify the trauma trigger at history taking which precipitated the OCD symptoms, it may be more helpful to start by targeting the past distressing memory first. It is the author's view that in Abigail's case, this may have alleviated her feelings of frustration at the lack of initial progress as expressed during treatment and her posttreatment qualitative interview. If this is the route taken, care must be taken to ensure that all current triggers are worked through to allow any other subsequent trauma memories to arise.

\section{The Role of Shame}

The role of shame in OCD is well documented. Researchers have postulated that both shame and guilt are emotions important to OCD (Fergus, Valentiner, McGrath, \& Jenicus, 2010; Weingarden \& Renshaw, 2014). Shame can have several consequences. On average, a person with OCD lives with the condition for 10 years before seeking help (Veale \& Wilson, 2005). All three case studies illustrate this, seeking help after 10 years, $10-15$ years, and 30 years, respectively (although Abigail did have some therapy with the child and adolescent mental health service aged 12 years). Shame can also mean that OCD is not discussed with family, friends, or partners, increasing the sense of isolation, not getting to hear that intrusive thoughts and doubts are "normal," and closing down support options. Again, all case studies illustrate this. It is also not unusual for clients with OCD to struggle to talk to health professionals about their OCD.

So what can EMDR offer? Shapiro (2001) advised that disclosing information is not crucial to the success of EMDR.

Sometimes a client is unwilling to concentrate on a particular memory because of shame or guilt. The clinician should reassure the client 
that because processing is happening internally, she need not divulge the details of the memory; merely reporting the fact she is withholding something is sufficient. (Shapiro, 2001, p. 132)

A modification to the standard EMDR protocol, the B2T protocol (Blore \& Holmshaw, 2009) was developed to bypass client unwillingness to describe trauma material and has been used most often in practice with issues of shame and guilt (Blore, Holmshaw, Swift, Standart, \& Fish, 2013). With Jan, the B2T protocol was used with a distressing past-related memory. The B2T protocol could also be used in the current triggers and future template phases of the OCD treatment. This is reliant on the client being able to inform the therapist that there are obsessions and compulsions that he or she cannot talk about. Explaining this option in the preparatory phases of EMDR, being able to work internally without divulging the material, may be one way to address the significant role that shame plays in OCD. Also, regarding assessing suitability for different treatment options, struggling to talk about OCD symptoms may indicate EMDR as a more helpful option than the standard CBT/ERP approach.

\section{Therapeutic Alliance}

It is easy to forget about the importance of the therapeutic alliance in deference to the treatment approach, perhaps particularly so when working with a new treatment protocol and within the dictates of a research trial. Norcross (2010) describes the therapeutic alliance as the most important predictor of treatment success and is the container for the treatment model.

The importance of the therapeutic alliance in EMDR therapy has been studied in other qualitative and mixed methods studies. For example, Marich (2010) highlights this as an important factor for all the participants in this qualitative study of women recovering from addiction. In contrast, Edmond, Sloan, and McCarty's (2004) study of sexual abuse survivors' perceptions of the effectiveness of EMDR, the participants attributed success to the "technical" EMDR process rather than the therapeutic alliance.

Francine Shapiro said,

The clinician must establish a relationship with the client that includes firm therapeutic alliance, a recognition of common goals and an understanding of the need for honest communication. Unless the client and clinician have established a sufficient level of trust, EMDR should not be used. Clients must feel that they will be protected during processing and that, ultimately, they are in control. (Shapiro, 2001, p. 122)

Feeling safe in therapy can be a primary concern. People feel safe when they know what to expect and what to do. This was of particular concern to the therapist because participating in a research trial with a previously untested treatment is taking a risk. Care was taken therefore to establish structure and routines, so the clients would know what to expect and do. In the participant feedback, Sarah described "feeling safe" in treatment and Jan was able to disclose that she was unable to talk about a past distressing memory and subsequently processed this.

Shapiro (2001) states that the client needs to feel in control during EMDR. Marr's (2012) Adapted EMDR Phobia Protocol enables participants to be active in the process, with it being a client decision, which order the current triggers (OCD thoughts and behaviors) and future templates are worked through in treatment.

\section{Conclusion}

This case series reflects the experience of one therapist and three clients who took part in a clinical trial applying an EMDR protocol (Marr, 2012) for the treatment of OCD symptoms. There are several strengths that support the credibility of this article, such as the random assignment of cases, the requirement of treatment fidelity as part of a clinical trial, the inclusion of the therapist and patient perspectives, the inclusion of validated psychometric measures, and the independent posttreatment qualitative interviews carried out by another researcher. Some caveats and limitations to note include the small number of cases reviewed and the fact that the cases in this article may not be a representative of more complex or severe OCD cases that tend to be screened out of primary care settings. Overall, the treatment seemed to work well with two cases at 6-month follow-up, reducing symptoms below the diagnostic cutoff on the validated psychometric measures and having more than a $50 \%$ reduction in symptoms. The final case started treatment below diagnostic cutoff on the primary measure and showed only slight improvement.

\section{References}

Abramowitz, J. S. (1997). Effectiveness of psychological and pharmacological treatments for obsessive-compulsive disorder: A quantitative review. Journal of Consulting and Clinical Psychology, 65(1), 44-52.

Abramowitz, J. S. (1998). Does cognitive-behavioral therapy cure obsessive-compulsive disorder? A meta-analytic 
evaluation of clinical significance. Behavior Therapy, 29, 339-355.

Aderka, I. M., Anholt, G. E., van Balkom, A. J., Smit, J. H., Hermesh, H., Hofmann, S. G., \& van Oppen, P. (2011). Differences between early and late drop-outs from treatment for obsessive-compulsive disorder. Journal of Anxiety Disorders, 25, 918-923.

American Psychiatric Association. (2013). Diagnostic and statistical manual of mental disorders (5th ed.). Washington, DC: Author.

Blore, D. C., \& Holmshaw, M. (2009). EMDR "blind to therapist protocol.” In M. Luber (Ed.), Eye movement desensitization and reprocessing: EMDR scripted protocols basic and special situations (pp. 233-240). New York, NY: Springer Publishing.

Blore, D. C., Holmshaw, E. M., Swift, A., Standart, S., \& Fish, D. M. (2013). The development and uses of the "blind to therapist" EMDR protocol. Journal of EMDR Practice and Research, 7(2), 95-105.

Bohm, K., \& Volderholzer, U. (2010). Use of EMDR in the treatment of obsessive-compulsive disorder: A case series. Verhaltenstherapie, 20, 175-181.

Braun, V., \& Clarke, V. (2006). Using thematic analysis in psychology. Qualitative Research in Psychology, 3, 77-101.

Cromer, K. R., Schmidt, N. B., \& Murphy, D. L. (2007). An investigation of traumatic life events and obsessivecompulsive disorder. Behaviour Research and Therapy, 45(7), 1683-1691.

Delgadillo, J., Marsden, Z., Lovell, K., Blore, D., \& Ali, S. (2014). Research Protocol: The ECO Trial (EMDR vs CBT in OCD). REC Reference No. 13/YH/ 0338 (27.2.14-v5). Retrieve from http://www.emdryorkshire.org/resource/ john-marr.pdf

De Silva, P., \& Marks, M. (1999). The role of traumatic experiences in the genesis of obsessive-compulsive disorder. Behaviour Research and Therapy, 37, 941-951.

Edmond, T., Sloan, L., \& McCarty, D. (2004). Sexual abuse survivors' perceptions of the effectiveness of EMDR and eclectic therapy. Research on Social Work Practice, 14(4), 259-272.

Fergus, T. A., Valentiner, D. P., McGrath, P. B., \& Jenicus, S. (2010). Shame- and guilt-proneness: Relationships with anxiety disorder symptoms in a clinical sample. Journal of Anxiety Disorders, 24, 811-815.

Fisher, P. L., \& Wells, A. (2005). How effective are cognitive and behavioral treatments for obsessive-compulsive disorder? A clinical significance analysis. Behaviour Research and Therapy, 43, 1543-1558.

Foa, E. B., Kozak, M. J., Salkovskis, P. M., Coles, M. E., \& Amir, N. (1998). The validation of a new obsessivecompulsive disorder scale: The Obsessive-Compulsive Inventory. Psychological Assessment, 10, 206-214.

Foa, E. (2010). Cognitive behavioral therapy of obsessivecompulsive disorder. Dialogues in Clinical Neuroscience, 12(2), 199-207.

Fostick, L., Nacasch, N., \& Zohar, J. (2012). Acute obsessive compulsive disorder (OCD) in veterans with posttraumatic stress disorder (PTSD). The World Journal of Biological Psychiatry, 13, 312-315.

Franklin, M., \& Foa, E. (2002). Cognitive behavioural treatments for obsessive compulsive disorder. In P. E. Nathan, J. M. Gorman (Eds.), A guide to treatments that work (pp. 367-386). New York, NY: Oxford University Press.

Gerger, H., Munder, T., Gemperli, A., Nuesch, E., Trelle, S., Jüni, P., \& Barth, J. (2014). Integrating fragmented evidence by network meta-analysis: Relative effectiveness of psychological interventions for adults with posttraumatic stress disorder. Psychological Medicine, 44(15), 3151-3164.

Goodman, W. K., Price L. H., Rasmussen, S. A., Mazure, C., Fleishmann, R. L., Hill, C. L., . . . Charney, D. S. (1989). The Yale-Brown Obsessive Compulsive Scale. I. Development, use and reliability. Archives of General Psychiatry, 46(11), 1006-1011.

Keenan, P., Keenan, L., Ingham, C., \& Farrell, D. (2014, June). Treating obsessive compulsive disorder (OCD) using eye movement desensitisation and reprocessing (EMDR): A case series design. In EMDR Research Symposium. Symposium conducted at 15th EMDR Europe Association Conference, Edinburgh, Scotland.

Kessler, R. C., Berglund, P., Demler, O., Jin, R., Merkangas, K. R., \& Walters, E. E. (2005). Lifetime prevalence and age-of-onset distributions of DSM-IV disorders in the National Comorbidity Survey Replication. Archives of General Psychiatry, 62(6), 593-602.

Improving Access to Psychological Therapies. (2011). The IAPT Data Handbook: Guidance on recording and monitoring outcomes to support local evidence-based practice. Version 2.0. London, United Kingdom: Author.

Logie, R., \& de Jongh, A. (2014). The "Flashforward procedure": Confronting the catastrophe. Journal of EMDR Practice and Research, 8, 25-32.

Maher, M. J., Huppert, J. D., Chen, H., Duan, N., Foa, E. B., Liebowitz, M. R., \& Simpson, H. B. (2010). Moderators and predictors of response to cognitive-behavioral therapy augmentation of pharmacotherapy in obsessive-compulsive disorder. Psychological Medicine, 40(12), 2013-2023.

Marich, J. (2010). Eye movement desensitization and reprocessing in addiction continuing care: A phenomenological study of women in recovery. Psychology of Addictive Behaviors, 24(3), 498-507.

Marr, J. (2012). EMDR treatment of obsessive-compulsive disorder: Preliminary research. Journal of EMDR Practice and Research, 6(1), 2-15.

Meyer, V. (1966). Modification of expectations in cases with obsessional rituals. Behaviour Research and Therapy, 4(4), 273-280.

Nazari, H., Momeni, N., Jariani, M., \& Tarrahi, M. J. (2011). Comparison of eye movement desensitization and reprocessing with citalopram in treatment of obsessivecompulsive disorder. International Journal of Psychiatry in Clinical Practice, 15, 270-274. 
Norcross, J. C. (2010). The therapeutic relationship. In B. L. Duncan, S. D. Miller, B. E Wampold, \& M. A. Hubble (Eds.), The heart and soul of change: Delivering what works in therapy (2nd ed., pp. 113-141). Washington, DC: American Psychological Association.

Parnell, L. (2007). A therapist's guide to EMDR: Tools and techniques for successful treatment. New York, NY: Norton.

Rosso, G., Albert, U., Asinari, G. F., Bogetto, F., \& Maina, G. (2012). Stressful life events and obsessive-compulsive disorder: Clinical features and symptom dimensions. Psychiatry Research, 197(3), 259-264.

Shapiro, F. (2001). Eye movement desensitization and reprocessing: Basic principles, protocols, and procedures. New York, NY: Guilford Press.

Shapiro, F., \& Forrest, M. (2004). EMDR: The breakthrough therapy for overcoming anxiety, stress and trauma. New York, NY: Perseus Books.

Sheenan, D., Lecrubier, Y., Sheenan, H., Amorin, P., Janavs, J., Weiller, E., . . Dunbar, G. (1998). The Mini-International Neuropsychiatric Interview (M.I.N.I): The development and validation of a structured diagnostic psychiatric interview for DSM-IV and ICD-10. Journal of Clinical Psychiatry, 59(Suppl. 20), 22-23.

Tolin, D. F., Abramowitz, J. S., \& Diefenbach, G. J. (2005) Defining response in clinical trials for obsessive compulsive disorder: A single detection analysis of the Yale-Brown obsessive compulsive scale. The Journal of Clinical Psychiatry, 66(12), 1549-1557.

Veale, D., \& Wilson, R. (2005). Overcoming obsessive compulsive disorder: A self help guide using cognitive behavioural techniques. London, United Kingdom: Robinson.

Watts, B. V., Schnurr, P. P., Mayo, L., Young-Xu, Y., Weeks, W. B., \& Friedman, M. J. (2013). Meta-analysis of the efficacy of treatments for posttraumatic stress disorder. The Journal of Clinical Psychology, 74, e541-e550.

Weingarden, H., \& Renshaw, K. D. (2014). Associations of obsessive compulsive symptoms and beliefs with depression: Testing mediation by shame and guilt. International Journal of Cognitive Therapy, 7(4), 305-319.

Acknowledgments. The author expresses appreciation to Jaime Delgadillo, Alex Teehan, David Blore, and everyone involved in the ECO trial for their help and support.

Correspondence regarding this article should be directed to Zoe Marsden, Leeds IAPT, Leeds Community Healthcare, Senior Mental Health Practitioner, Burmantofts Health Centre, Cromwell Mount, Leeds LS9 7TA. E-mail: zoe.marsden@nhs.net 